\title{
molecules
}

ISSN 1420-3049

http://www.mdpi.org/

\section{Determination of Aldehyde Dehydrogenase (ALDH) Isozymes in Human Cancer Samples - Comparison of Kinetic and Immunochemical Assays}

Piotr Wroczynski ${ }^{1 *}$, Jacek Wierzchowski ${ }^{2}$, Jakub Golab $^{3}$, Magdalena Blazejczyk ${ }^{1}$ and Dorota Borecka $^{1}$

${ }^{1}$ Laboratory of Organic Chemistry, Faculty of Pharmacy and Department of Immunology, Medical University, 1 Banacha St., Warsaw, PL-02097, Poland.

${ }^{2}$ Institute of Chemistry, University of Podlasie, 54 Trzeciego Maja St., Siedlce, PL-08-110, Poland; e-mail: jacekwie@acn.waw.pl.

${ }^{3}$ Department of Immunology, Medical University, 2 Oczki St., Warsaw, PL-00-052, Poland.

* Author to whom correspondence should be addressed; e-mail: mailto:wropio@farm.amwaw.edu.pl Received: 25 October 2002 / Accepted: 4 December 2002 / Published: 31 December 2002

\begin{abstract}
A fluorimetric assay of aldehyde dehydrogenase isozymes, based on naphthaldehyde oxidation, is compared with Western Blotting analysis on several clinical samples obtained from surgery. The comparison reveals qualitatively good correlation of ALDH1A1 isozyme detection with two methods and somewhat worse on ALDH3A1 assay.
\end{abstract}

Keywords: Aldehyde dehydrogenase, isozymes, western blotting, fluorimetric assays

\section{Introduction}

A ubiquitous, polymorphic enzyme aldehyde dehydrogenase (ALDH, E.C.1.2.1.3) plays an important role in the process of inactivation of many xenobiotics, including anti-cancer drugs of the oxazaphosphoprine series, in living cells [1,2]. It has been confirmed that two cytosolic ALDH isozymes, ALDH1A1 and ALDH3A1 (formerly known as ALDH-1 and ALDH-3) are those primarily responsible for drug inactivation 
[2-4]. The activity levels of the foregoing isozymes also reveal some predictive potential for cancer metastasis level [5].

Evaluation of ALDH activity levels in clinical material by traditional spectrophotometric approach, i.e., using either acetaldehyde or propionic aldehyde as a substrate, and measuring NADH production rate, is not isozyme-specific and usually requires prior separation of the enzymes from the clinical sample. Much better specificity is obtained with immunochemical methods, primarily ELISA [6], evaluating protein content, rather than its activity. It has also been postulated that a novel fluorimetric assay, based on oxidation of fluorescent and fluorogenic naphthaldehydes [7,8], exhibits much better selectivity for the cytosolic ALDH forms, and therefore may be applied directly for crude tissue homogenates. The principle of the fluorimetric assay is shown on Scheme 1, below.<smiles>COc1ccc2cccc(C=O)c2c1</smiles>

7-methoxy-1-naphthaldehyde MONAL-71

\section{Scheme 1.}

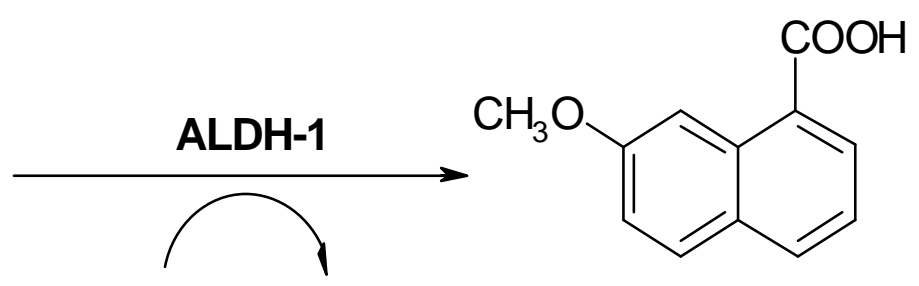

NAD+ NADH

7-methoxy-1-naphthoic acid MONCO-71

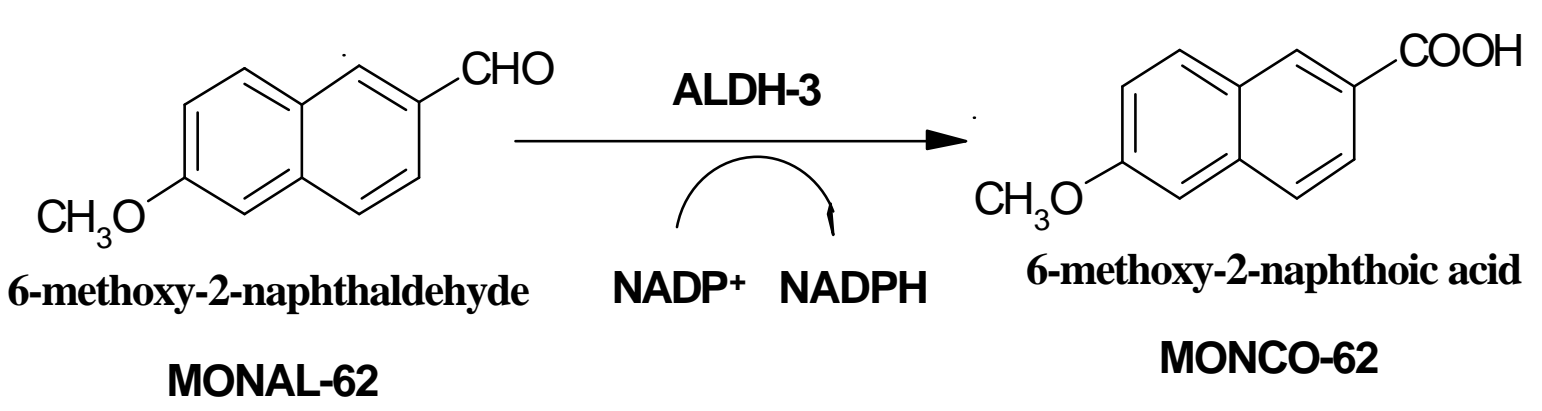

In the present paper, we compare results of ALDH isozyme level evaluation by two methods - fluorimetric and western blotting analysis in several clinical samples, obtained from surgery. The results reveal qualitatively good correlation. 


\section{Results and Discussion}

Five samples of colon tumours, two samples of liver cancers and one of stomach tumour, and the adjacent fragments of apparently healthy tissue, all excised surgically, were homogenized within 6 hrs after operation and ALDH isozymes activities analyzed fluorimetrically, as previously described [8]. The same samples, after freezing, were subjected to analysis by Western Blotting. The results are summarized in Table 1 and Fig. 1.

Table 1. Specific activities of ALDH isozymes, in units per gram protein, determined in tumour samples, compared to those found in the surrounded tissue (in parentheses), and to Western Blot analysis.

\begin{tabular}{l|cccc}
\hline \multirow{2}{*}{ Sample } & \multicolumn{2}{|c}{ Fluorimetric analysis } & \multicolumn{2}{c}{ Western Blot analysis } \\
& ALDH1A1 & ALDH3A1 & ALDH1A1 & ALDH3A1 \\
\hline Colon tumour 1 & $0.14(0.06)$ & $0.07(0.02)$ & $++(+)$ & $\pm( \pm)$ \\
Colon tumour 2 & $0.09(0.08)$ & $0.05^{\mathrm{a}}(0.10)$ & $+(+)$ & $-(+)$ \\
Colon tumour 3 & $0.21(0.15)$ & $0.17\left(0.1^{\mathrm{a}}\right)$ & $++(+)$ & $+( \pm)$ \\
Colon tumour 4 & $0.13(0.38)$ & $0.01^{\mathrm{a}}(0.05)$ & $+(++)$ & $\pm(++)$ \\
Colon tumour 5 & $0.07(0.08)$ & $0.04(0.07)$ & $++(++)$ & $++(++)$ \\
Liver cancer 6 & $0.14(1.4)$ & $1.61(1.61)$ & $-(++)$ & $+(+)$ \\
Liver cancer 7 & $0.19(2.5)$ & $0.51(0.66)$ & $+(++)$ & $+++(+++)$ \\
Stomach tumour 8 & $0.28(0.73)$ & $0.18(1.75)$ & $+(++)$ & $+(++)$ \\
\hline \multicolumn{5}{c}{}
\end{tabular}

The ALDH1A1 isozyme is expressed in nearly every human tissue, including blood, but its activity is variable [1,2], while ALDH3A1 is known to be inducible. The fluorimetric assays, showing high activity of ALDH1A1 in the liver, and much lower, but measurable, in the stomach and colon tissue, is in agreement with previous reports $[2,8,9]$. The naphthaldehyde activities, reported in this paper, are comparable to the more commonly used acetaldehyde activities, if acetaldehyde concentrations do not excede $200 \mu \mathrm{M}$ [7]. Differences between tumour and tissue activities have also been reported for other types of cancer [5,6] and may have both diagnostic and therapeutic significance [2].

There is a good qualitative correlation of Western Blot analysis and the results of activity measurement, particularly for ALDH1A1 isozyme in colon tissue. Somewhat higher variability of the activity measurements can be explained by high susceptibility of this isozyme to inactivation by drugs, alcohol, and other factors [10]. Within the colon samples, there is also quite good correlation of ALDH3A1 determinations by two methods, but this correlation is much worse for other types of tissue. This might be due to the polymorphism of ALDH3 type (dimeric) inducible isozymes [1]. 
Figure 1. Western blot analysis - tumour samples and the surrounded tissue (in parentheses)

$$
\begin{array}{llllllll}
1 & (1) & 2 & (2) & 3 & \text { (3) } 4 & \text { (4) } & 5
\end{array}
$$

\section{ALDH1A1}

ALDH3A1

\section{TUBULIN}

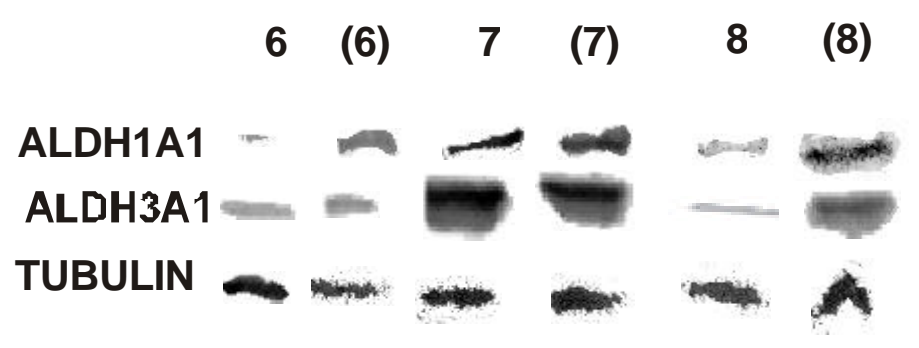

\section{Conclusions}

We have presented evidence confirming the specificity of the fluorimetric ALDH assay, utilizing 7-methoxy1-naphthaldehyde as a substrate, toward the cytosolic ALDH1A1 isozyme in the clinical samples, by showing correlation between fluorimetric and Western Blot analyses. Somewhat worse, but still satisfactory specificity is found for the fluorimetric ALDH3A1 assay. The fluorimetric method can be used to rapid screening of ALDH activity in clinical material.

\section{Experimental}

\section{General}

Naphthaldehyde substrates and the corresponding carboxylates were synthesized as previously described [7], and all reagents were from Sigma or Aldrich. Water was filtered through a Milli-Q system (Millipore Corp., U.S.A). Clinical samples were obtained from Proessor J. Polanski, III Dept. of Surgery, Medical School, Warsaw, with permission from the Ethical Commitee.

\section{Western Blot Tests}

Samples of tumor tissue as well as control nonmalignant tissues obtained from the tumor patients were homogenized and sonicated in a RIPA buffer supplemented with protease inhibitor cocktail tablets (Roche Diagnostics GmbH, Mannheim, Germany), sodium orthovanadate and PMSF. Protein concentration was 
measured with the use of BCA protein assay (Pierce, Rockford, IL, USA). Equal amounts of proteins were separated on $12.5 \%$ SDS-polyacrylamide gel, transferred onto PVDF membranes, blocked with TBST (Tris buffered saline $\mathrm{pH} 7.4,0.05 \%$ Tween-20) with 5\% non-fat milk and 5\% FBS. The following primary anibodies kindly obtained from Dr. L. Sreerama (Department of Chemistry, 372 Mathematics and Sceince Center, St. Cloud, MN, USA) were used for the overnight incubation: chicken polyclonal anti-ALDH1A1 at 1:500 dilution and chicken polyclonal anti-ALDH3A1 at 1:200 dilution. Protein loading was verified with antitubulin antibodies (Sigma Chemical Co., St. Louis, MO, USA). After extensive washing the membranes were incubated for 60 minutes with the alkaline phosphatase-coupled secondary antibodies against chicken IgG (Sigma). The color reaction was developed using NBT (p-nitro-blue tetrazolium chloride) and BCIP (5bromo-4-chloro-3-indolyl phosphate) (Sigma).

\section{Fluorimetric procedure}

The fluorimetric assays of ALDH isozymes utilize highly fluorogenic naphthaldehyde substrates (see Scheme 1). In particular, 7-methoxy-1-naphthaldehyde (MONAL-71) and 6-methoxy-2-naphth-aldehyde (MONAL-62), acting with $\mathrm{NAD}^{+}$and $\mathrm{NADP}^{+}$as co-substrates, respectively, are used to measure selectively the activities of the cytosolic ALDH1A1 and ALDH3A1 forms [7]. Fluorimetric assays were run in $50 \mathrm{mM}$ pyrophosphate/ $\mathrm{HCl}$ buffer $\mathrm{pH} 8.1$, at $25{ }^{\circ} \mathrm{C}$, in the presence of $2 \mathrm{mM} \mathrm{DTT}, 0.5 \mathrm{mM}$ EDTA. The homogenates were diluted ca. 15 -fold, resulting in $\sim 0.1-2 \mathrm{mg} / \mathrm{mL}$ final protein contents in the cuvette. Typical substrate concentrations were $45 \mu \mathrm{M}$ for $\mathrm{ALDH}$ and $\sim 15 \mu \mathrm{M}$ for $\mathrm{ADH}$ assays. Coenzymes $\mathrm{NAD}^{+}$and $\mathrm{NADP}^{+}$(Sigma) were used in concentrations of 100 and $300 \mu \mathrm{M}$ to measure ALDH1A1 and ALDH3A1 activities, respectively. Fluorescence background drift, if any, was measured prior to coenzyme addition and subtracted from the final slope. Purified reaction product(s) at concentrations of $3-5 \mu \mathrm{M}$ were used as internal standards to obtain absolute reaction rates [7]. Sensitivity limits for reaction rates were typically $\sim 0.1$ $\mathrm{nM} / \mathrm{min}$, which corresponded to specific activities of $\sim 0.01 \mathrm{U} /$ gram protein [7,8]. Protein concentration in the homogenate was measured by the Bradford method.

\section{References and Notes}

1. Lindahl, R. Aldehyde dehydrogenases and their role in carcinogenesis Crit. Revs. Biochem. Mol. Biol., 1992, 27, 283-335.

2. Sladek, N. E. Aldehyde dehydrogenase-mediated cellular relative insensitivity to the oxazaphosphorines. Curr. Pharm. Des., 1999, 5, 607-625.

3. Moreb, J.; Schweder, M.; Suresh, A.; Zucali, J. R. Overexpression of the human aldehyde dehydrogenase class I results in increased resistance to 4-hydroperoxycyclophosphamide. Cancer Gene Therapy, 1996, 3, 24-30. 
4. Moreb, J. S., Maccow, C., Schweder, M., Hecomovich, J. Expression of antisense RNA to aldehyde dehydrogenase class- 1 sensitizes tumor cells to 4-hydroperoxycyclophosphamide in vitro. J. Pharmacol. Exper. Therap., 2000, 293, 390-396.

5. Sreerama L.; Sladek N. E. Primary breast tumour levels of suspected molecular determinants of cellular sensitivity...., Cancer Therap. Pharmacol., 2001, 47, 255-62.

6. Sreerama L.; Sladek N. E. Cellular levels of class 1 and class 3 aldehyde dehydrogenases and certain other drug-metabolizing enzymes in human breast malignancies. Clin Cancer Res., 1997, 3, 1901-14.

7. Wierzchowski J.; Wroczynski P.; Laszuk K.; Interewicz E. Fluorimetric detection of aldehyde dehydrogenase activity in human blood, saliva and organ biopsies, and kinetic differentiation between class I and class III isozymes. Anal. Biochem., 1997, 245, 69-78.

8. Wroczynski P.; Laskowska A.; Wierzchowski J.; Szubert A.; Polanski J.; Slowiaczek M. Aldehyde dehydrogenase isoenzymes in tumours - assay with possible prognostic value for oxazaphosphorine chemotherapy. Acta Biochim. Pol., 1998; 48, 33-45.

9. Yin S. J.; Liao C. S.; Lee Y. C.; Wu C. W.; Jao S. W. Genetic polymorphism and activities of human colon alcohol and aldehyde dehydrogenases: no gender and age differences. Alcoholism Clin. Exper. Res. 1994, 18, 1256-60.

10. Helander A. Aldehyde deydrogenase in blood: distribution, characteristics and possible use as marker of alcohol misuse. Alcohol \& Alcoholism, 1993, 28, 135-145.

Sample Availability: Available from the authors

(C) 2002 by MDPI (http://www.mdpi.org). Reproduction is permitted for noncommercial purposes. 